\title{
Evaluación clínica, microbiológica e inmuológica de la ozonoterapia en pacientes con bolsas periodontales moderadas-severas
}

\author{
RIPOLLES DE RAMÓN J* \\ COLMENERO RUIZ C ** \\ GALLUT RUIZ J*** \\ ZAERA LE GAL R**** \\ BASCONES MARTINEZ $\mathrm{A} * * * * *$
}

\begin{abstract}
Ripollés de Ramón J, Colmenero Ruiz C, Gallut Ruiz J, Zaera Le gal R, Bastones Martínez A. Evaluación clínica, microbiológica e inmunológica de la ozonoterapia en pacientes con bolsas periodontales moderadas-severas. Av Periodon Implantol. 2004; 16, 1: 63-72.
\end{abstract}

\section{RESUMEN}

INTRODUCCIÓN. En los últimos años se ha producido un progreso importante en el conocimiento de la enfermedad periodontal, desde su etiología multifactorial, la microbiología responsable, la respuesta inmunológica local, así como múltiples aspectos en relación con su epidemiología, etiopatogenia y su proceso evolutivo. Dado el mayor conocimiento en estos campos, se desarrollan nuevas técnicas, productos farmacológicos y técnicas que tratan de detener la enfermedad periodontal y preservar la salud bucal.

OBJETIVOS. Analizar las respuestas periodontales desde un punto de vista clínico, microbiológico e inmunológico de una población con bolsas periodontales moderadas severas y su comparación, tras la ozonoterapia, con la técnica del raspado y alisado radicular.

MATERIAL Y MÉTODO. Se estudiaron un total de 72 cuadrantes maxilares con al menos $>4$ dientes en dichos cuadrantes y $>6 \mathrm{~mm}$ de profundidad de sondaje (bolsa periodontal moderada-severa) en una población con una media de edad de 43 años. Para establecer las comparaciones entre ambos grupos se realizó un estudio a boca partida en el que la mitad de los cuadrantes fueron tratados con ozono y la otra restante con una técnica de raspado y alisado radicular. Se evaluarón las variables respuestas: clínico-microbiológico e inmunológico mediante análisis estadísticos descriptivo y ANOVA.

CONCLUSIÓn. Podemos establecer de forma preliminar, que el tratamiento periodontal con ozono $\left(\right.$ PrimoLog $\mathrm{O}_{3}$ ) produce una reducción estadísticamente significativa en el índice de sangrado gingival de Lindhe, en la microbiología patógena periodontal así como de los patrones inmunológicos de la Ikl-b y el TNF-alfa, sin embargo no produce variación alguna en relación al nivel de inserción periodontal y de la profundidad de la bolsa periodontal que requiere su remoción mecánica con el raspado y alisado radicular. Asimismo se requiere de un mayor numero de estudios que corroboren estos hallazgos.

Aceptado para publicación: Julio 2003

\section{PALABRAS CLAVE}

Ozono, Microbiología, Inmunología, Biooxidación, Periodontitis.

\footnotetext{
* Doctorando Departamento de Medicina y Cirugía Bucofacial. Facultad de Odontología. UCM. Madrid.

** Cirugía bucal. Práctica privada. Madrid. Coordinador área III. Imsalud.

*** Doctorando UPV.

**** Periodoncista. Universidad de París V. Práctica privada periodoncia e implantes. Zaragoza.

***** Catedrático de Medicina Bucal y Periodoncia. Departamento de Estomatología III. Facultad de Odontología UCM. Madrid.
} 


\section{INTRODUCCIÓN}

El oxígeno y los productos oxigenados son determinantes microbiológicos periodontales, puesto que influyen, de manera determinante en la capacidad bacteriana de reproducción y crecimiento, en función de distintas concentraciones de oxígeno. En relación con este espectro de tolerancia al oxígeno, la separación de las bacterias en aerobias y anaerobias es bastante arbitraria de tal forma que intracelularmente o en cualquier punto caracterizado por un potencial de oxidorreducción bajo, el oxígeno es convertido en productos altamente reactivos y potencialmente destructivos, siendo el oxígeno reducido, en pasos electrónicos, formándose aniones de superóxido $\left(\mathrm{O}^{2-}\right)$, Peróxido de Hidrógeno $\left(\mathrm{H}_{2} \mathrm{O}_{2}\right)$ y radicales oxhidrilos ${ }^{1-6}$. Con el fin de sobrevivir en un medio aerobio, las células bacterianas están equipadas con un complejo sistema multienzimático coordinado (peroxidasas, dismutasas y catalasas) que permiten la conversión de estos aniones oxidativos y celularmente lesivos en agua; de tal manera, que las bacterias anaerobias no presentan este mecanismo multienzimático regulador. El desarrollo microbiológico a nivel periodontal crea puntos con distintos niveles de oxígeno y potencial redox, siendo éstos muy bajos en la base de la bolsa periodontal ${ }^{6}$.

En base a los estudios de microbiología periodontal, inmunología y de la composición del fluido gingival crevicular (GCF) ${ }^{7-16}$, distintos autores, han presentado alternativas terapéuticas diferentes al tratamiento convencional del raspado y alisado radicular en las bolsas periodontales, dando lugar a nuevos productos farmacológicos y terapéuticos que han permitido un avance significativo en este tipo enfermedad. Estudios retrospectivos sobre terapias biooxidativas a nivel periodontal han demostrado que la actividad antimicrobiana de los agentes oxidantes entre ellos el ozono, es significativo en relación a los parámetros de: tiempo y concentración ${ }^{1,2,4,6}$.

Los estudios realizados en periodoncia con ozono, presentan este sustrato de diferentes formas: agua ozonizada, aceites ozonizados, ozono en gas, entre otras formas. El presente aparato (PrimoLogO ${ }_{3}$ de Kuss Dental) permite crear el ozono a través del propio oxígeno de la región tisular en la que se aplica por medio de corrientes de alta frecuencia, permitiendo así, la transformación del oxígeno circundante en ozono, impulsándolo a una distancia al menos de 12-15 mm respecto a su lugar de aplicación.

\section{OBJETIVOS DEL ESTUDIO}

El objetivo principal del estudio, se basa en la evalua- ción de la respuesta periodontal del paciente desde un punto de vista clínico (índice de sangrado, índice de higiene oral y profundidad de sondaje), microbiológico (estudio de principales periodontaopatógenos) e inmunológico (interleuquina lb y factor de necrosis tumoral TNF) al aplicar ozono frente a la técnica del raspado y alisado radicular.

\section{MATERIAL Y MÉTODOS}

\section{MUESTRA Y TIPO DE POBLACIÓN}

Estudiamos un grupo de pacientes que acudieron al Departamento de Estomatología III (Periodoncia y Medicina bucal) de la Facultad de Odontología de la UCM y que presentaron todos ellos un cuadro de enfermedad periodontal con bolsas periodontales moderadas-severas ( $>6 \mathrm{~mm}$ de profundidad al sondaje). Se estudiaron 72 cuadrantes maxilares de una población, con un rango de edad comprendido entre los 25 y 65 años. Se diseñó un estudio clínico prospectivo y randomizado a boca partida, en el que a cada cuadrante susceptible de estudio, se le asignó indistintamente una modalidad $\mathrm{u}$ otra de tratamiento. La población objeto de estudio presentó al menos dos de los cuatro cuadrantes con cuatro dientes con bolsas periodontales con una profundidad al sondaje superior a los $6 \mathrm{~mm}$ en al menos un punto de cada medición periodontal

\section{CARACTERÍSTICAS DE LA POBLACIÓN}

Se procedió a la selección de los sujetos objeto de estudio, en base a unos criterios de selección (criterios de inclusión/ criterios de exclusión).

\section{Criterios de inclusión. (Tabla 1 y Tabla 2)}

- Paciente adultos varones de $70 \mathrm{Kg}$ de peso y mujeres de $65 \mathrm{Kg}$. con una desviación no superior a un $15 \%$ en cuanto al peso en ambos sexos.

- Presencia de al menos cuatro dientes con bolsas periodontales superiores a $6 \mathrm{~mm}$ de profundidad de sondaje en al menos dos cuadrantes maxilares. (al menos un punto de cada medición periodontal presentaba dicha medición).

- No ingesta de antibióticos en las últimas seis semanas anteriores al estudio.

- Presencia de salud general con ausencia de enfermedades generales que influyan en la evolución de la enfermedad periodontal

- Ausencia de experiencia frente al tratamiento periodontal.

- Pacientes que acuden al Departamento de Medicina y Cirugía Bucofacial de la Facultad de Odontología de la U.C.M. como primera visita a tratamiento periodontal. 


\begin{tabular}{|lccccc|}
\hline \multicolumn{5}{|c|}{ TABLA } & 1.- DESCRIPCIÓN ANALITICA DE LA MUESTRA POBLACIONAL \\
& GRUPO CONTROL & \\
\hline & MEDIA & D.s. & MODA & RANGO & P50 \\
\hline Edad (años) & 48,50 & 2,72 & $62(3)$ & $64-25$ & 49,30 \\
Peso (kg) & 64,39 & 2,99 & - & $78-52$ & 54,50 \\
\hline
\end{tabular}

\begin{tabular}{|lccccc|}
\hline \multicolumn{5}{|c|}{ TABLA 2.- DESCRIPCIÓN ANALÍTICA DE LA MUESTRA POBLACIONAL } \\
GRUPO EXPERIMENTAL \\
\hline \\
\cline { 2 - 6 } & MEDIA & D.s. & MODA & RANGO & P50 \\
\hline Edad (años) & 42,74 & 2,44 & - & $65-28$ & 47,00 \\
Peso (kg) & 67,29 & 3,05 & - & $79-56$ & 56,20 \\
\hline
\end{tabular}

Criterios de exclusión.

- Mujeres embarazadas o en periodos de lactancia.

- Enfermedades sistémicas que puedan interferir en la evolución de la patología.

- Aquellos pacientes que no puedan cumplir las visitas objeto de nuestro estudio.

\section{DESARROLLOY MÉTODO DEL ENSAYO}

En una primera visita se procedió a la verificación de los criterios de selección, así como de los procedimientos operatorios que incluían nuestro protocolo de actuación.

Tras un análisis médico y dental pormenorizado y tras cumplir los requisitos necesarios para su inclusión se procedió a aceptar las condiciones necesarias y su consentimiento informado pertinente.

Inicio fase experimental.
Se procedió a la valoración de ciertas variables clínicas, microbiológicas e inmunológicas en una situación basal.

\section{Variables clínicas: (Tablas 3 y 4 )}

Presencia/Ausencia de placa supragingival (Figural): Indice de O'Leary y Lindhe (HI) ${ }^{14-18}$ Valoración de la gingivitis y severidad de la enfermedad periodontal mediante el índice PI de Russell ${ }^{1417}$.

Material: Se utilizó eritrosina al 3\% de Henry-Schein para la valoración del índice de higiene. La profundidad de sondaje fue medida por un único operador y a través de una sonda periodontal Hu-Friedy CPll.

Variables microbiológicas.

Periodontopatógenos principales (Figura 2) ( $\mathrm{AC}$ tinobacillus actinomicetemcomitans, Bacteroides forsythus, Porphyromonas gingivalis, entre otros) $)^{14,17,18,20,21-4}$.

Recuento de flora patógena total (Figuras 3 y 4) (UFO).

\begin{tabular}{|lrcccc|}
\hline \multicolumn{5}{|c|}{ TABLA 3.- DESCRIPCIÓN ANALITICA DE LAS VARIABLES PRINCIPALES } \\
EN EL GRUPO CONTROL & & \\
\hline & MEDIA & D.s. & MODA & RANGO & P50 \\
\hline ISG-inicial & 64,33 & 2,95 & - & $75,12-49,12$ & 63,33 \\
IAG-final & 29,45 & 2,13 & - & $42,12-21,40$ & 30,23 \\
PI-inicial & 05,94 & 0,93 & $4,2(2)$ & $07,20-04,31$ & 04,98 \\
PI-final & 01,83 & 0,50 & - & $02,21-01,40$ & 01,93 \\
Flora bact (10x5)-I & 299,00 & 6,51 & - & $722-78$ & 252,00 \\
Flora bact (10x5)-f & 192,00 & 5,16 & - & $177-40,5$ & 173,00 \\
HI-inicial & 31,28 & 2,10 & - & $38,12-020,12$ & 32,12 \\
HI-final & 47,21 & 2,62 & - & $74,12-30,20$ & 43,99 \\
TNF-a-inicial & 90,12 & - & - & $154-45,20$ & 74,23 \\
TNF-a-final & 48,99 & - & - & $72,14-23,12$ & 45,90 \\
Hl-1b-inicial & 82,33 & - & - & $134,89-64,89$ & 77,23 \\
Hl-1b-final & 42,33 & - & - & $78,14-29-21$ & 41,30 \\
\hline
\end{tabular}




\begin{tabular}{|lrlccr|}
\hline \multicolumn{5}{|c|}{ TABLA 4.- DESCRIPCIÓN ANALITICA DE LAS VARIABLES PRINCIPALES } \\
& EN EL GRUPO EXPERIMENTAL & & \\
\hline & MEDIA & D.s. & MODA & RANGO & P50 \\
\hline ISG-inicial & 62,43 & 2,97 & $72,12(2)$ & $72,12-46,36$ & 63,59 \\
IAG-final & 26,29 & 1,98 & $29,15(2)$ & $32,34-15,12$ & 28,33 \\
PI-inicial & 5,72 & 0,90 & - & $7,60-4,62$ & 5,09 \\
PI-final & 5,59 & 0,89 & - & $6,99-4,80$ & 5,83 \\
Flora bact (10x5)-I & 256,77 & 5,98 & - & $493-20,8$ & 330,00 \\
Flora bact (10x5)-f & 125,00 & 4,15 & - & $336-4,16$ & 178,00 \\
HI-inicial & 31,30 & 2,14 & - & $42,01-15,25$ & 31,72 \\
HI-final & 49,99 & 2,45 & - & $80,5-24,15$ & 49,12 \\
TNF-a-inicial & 101,23 & - & - & $42,21-125,25$ & 97,99 \\
TNF-a-final & 36,01 & - & - & $70,5-23,15$ & 30,12 \\
Hl-1b-inicial & 96,12 & - & - & $144-45,39$ & 84,27 \\
Hl-1b-final & 34,28 & - & - & $71,14-23,09$ & 32,67 \\
\hline
\end{tabular}

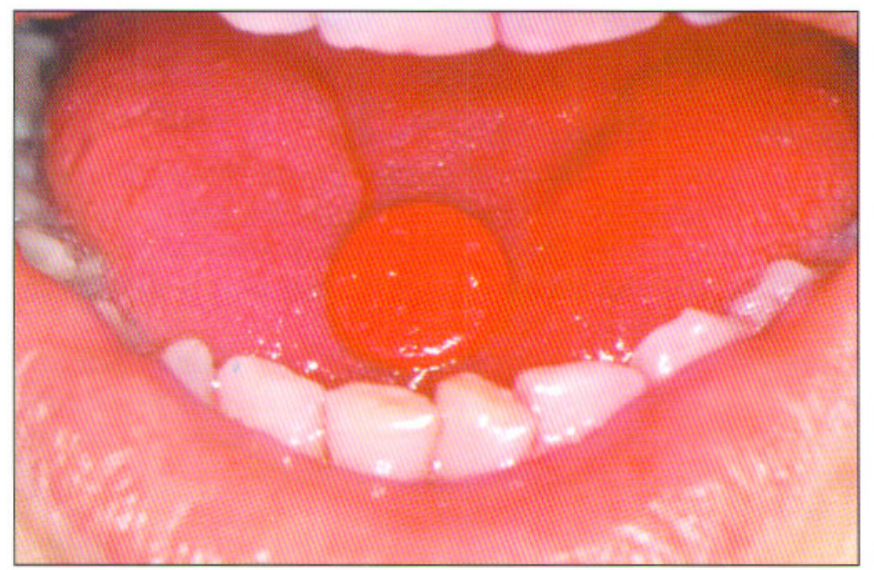

Imagen 1. Medición del índice de placa con eritrosina 3\%.

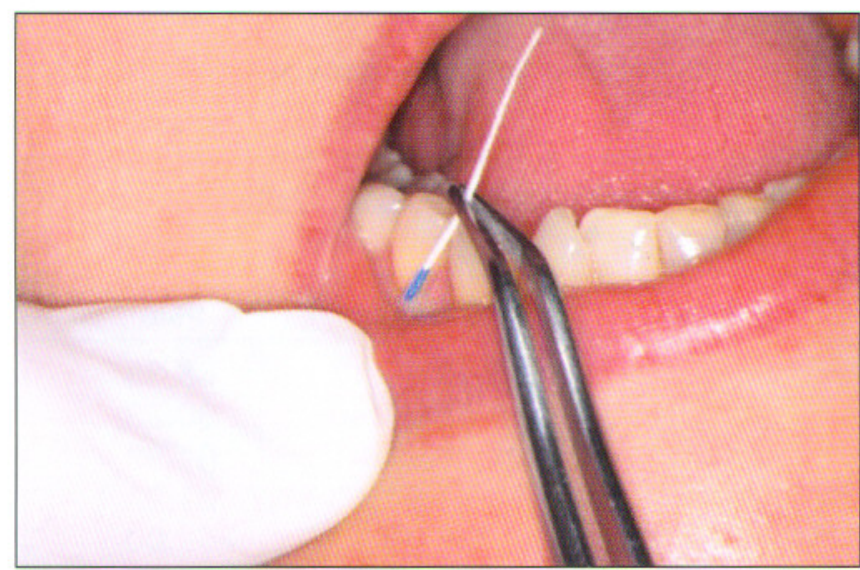

Imagen 2. Análisis microbiológico periodontal. ISO 30.

Material: Se utilizaron puntas de papel estandarizadas ISO 30 ( 8 puntas por muestra) más 2 puntas por localización periodontal y $2 \mathrm{ml}$. De RTF como medio de transporte.

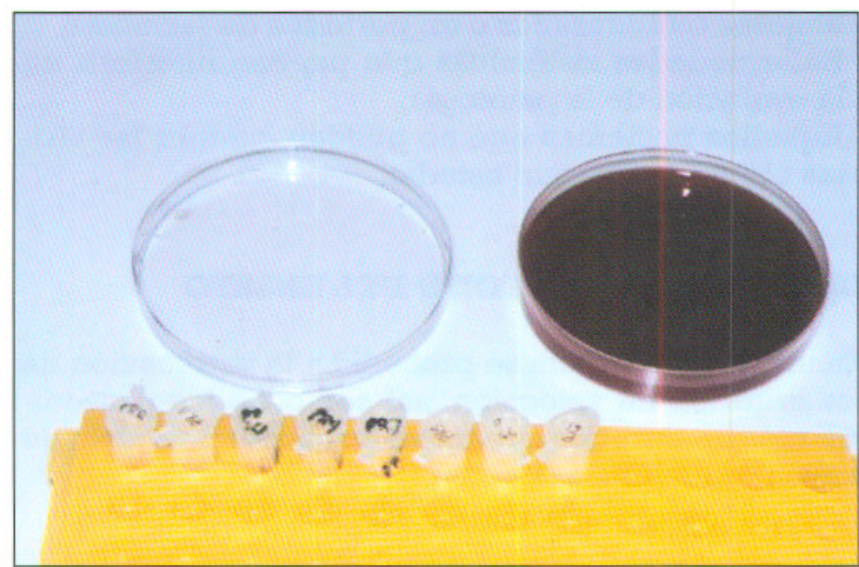

Imagen 3. Recuento microbiológico.

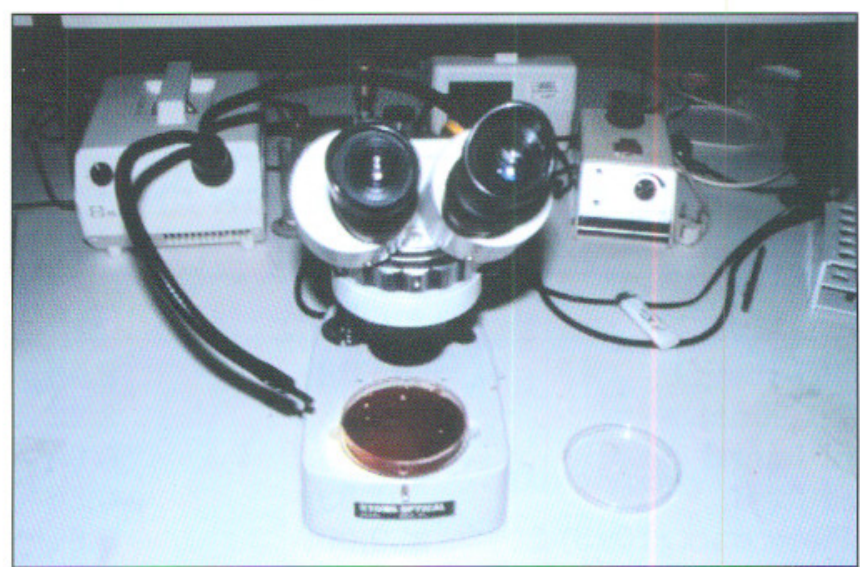

Imagen 4. Recuento microbiológico. Laboratorio Microbiología UCM.

Variables inmunológicas.(Tablas 3 y 4)

Interleuquina $1 \mathrm{~b} 25-31$

Factor de necrosis tumoral alfa (FNT-alfa). 


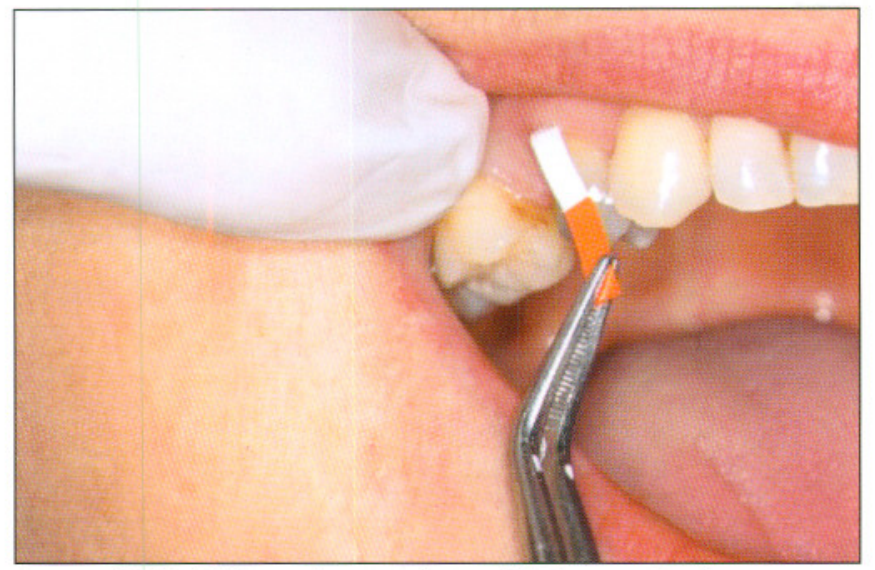

Imagen 5. Medición parámetros inmunológicos con Periopaper.

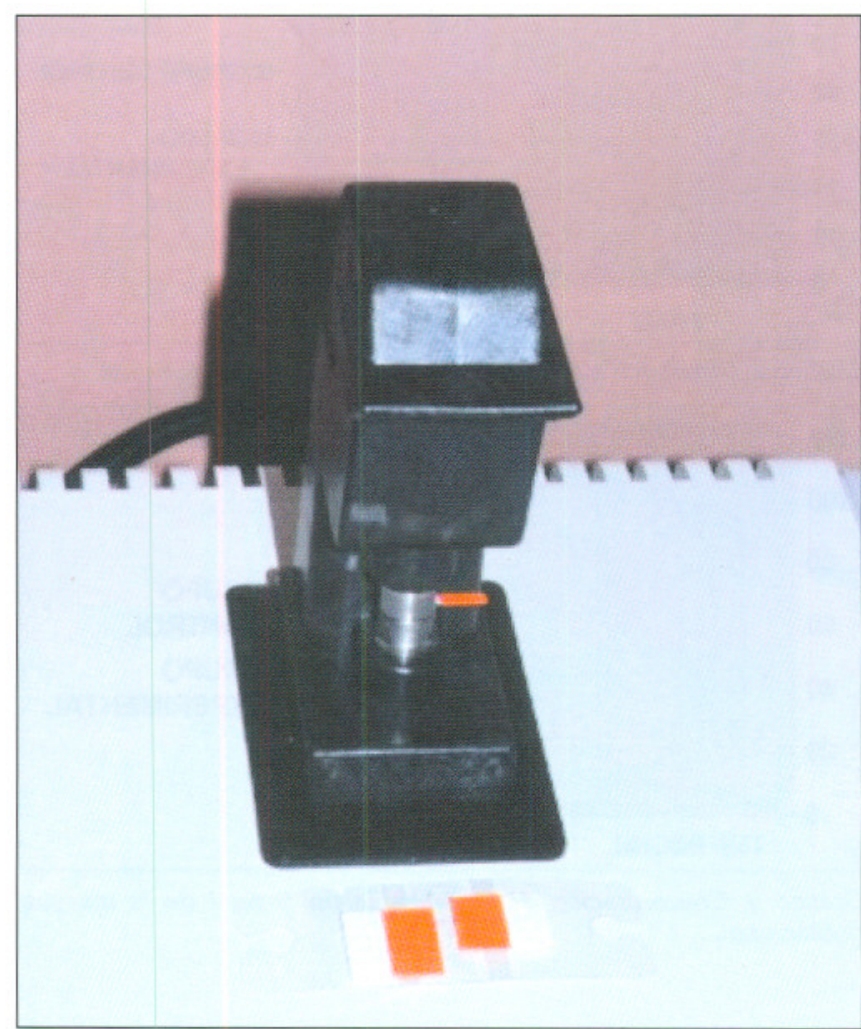

Imagen 6. Medición con Periotron de FGC del Periopaper.

Material: Tiras de papel absorbente: Periopaper. (Figuras 5 y 6 ))

Kit enzimático de inmunoensayo IL-1b IMMUNOTECH

Test de ELISA para el factor de necrosis tumoral-alfa.

\section{Aplicación del ozono en cuadrantes experimentales.}

En el cuadrante escogido de forma aleatoria para la

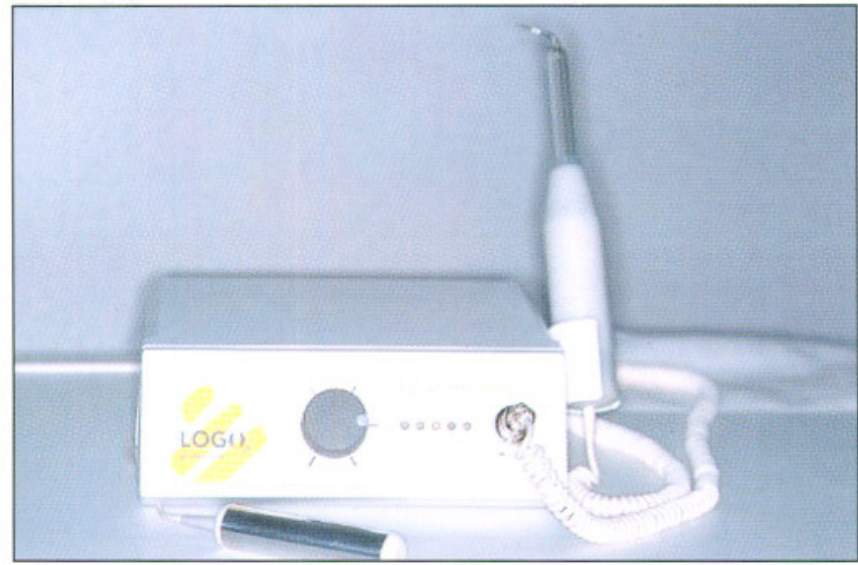

Imagen 7. PrimoLogO $\mathrm{O}_{3}$ de Kuss Dental.

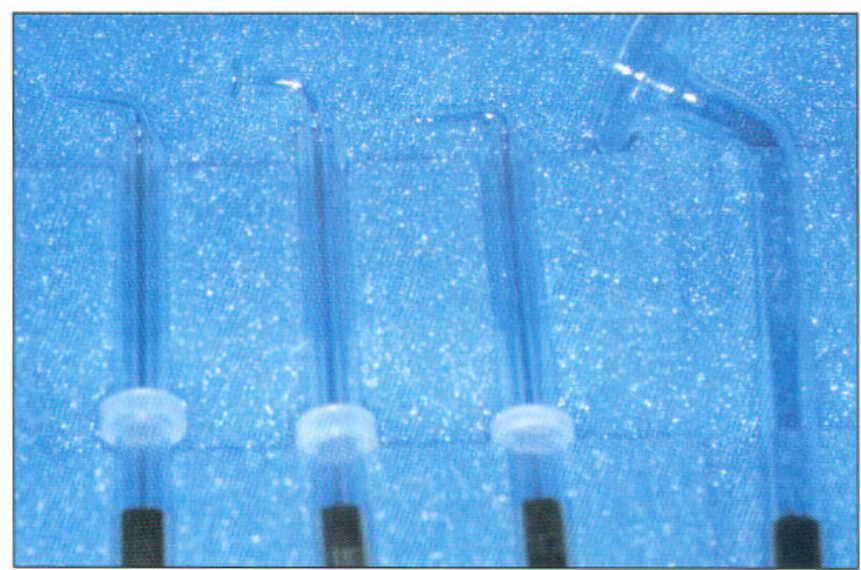

Imagen 8. Sondas de aplicación del ozono.

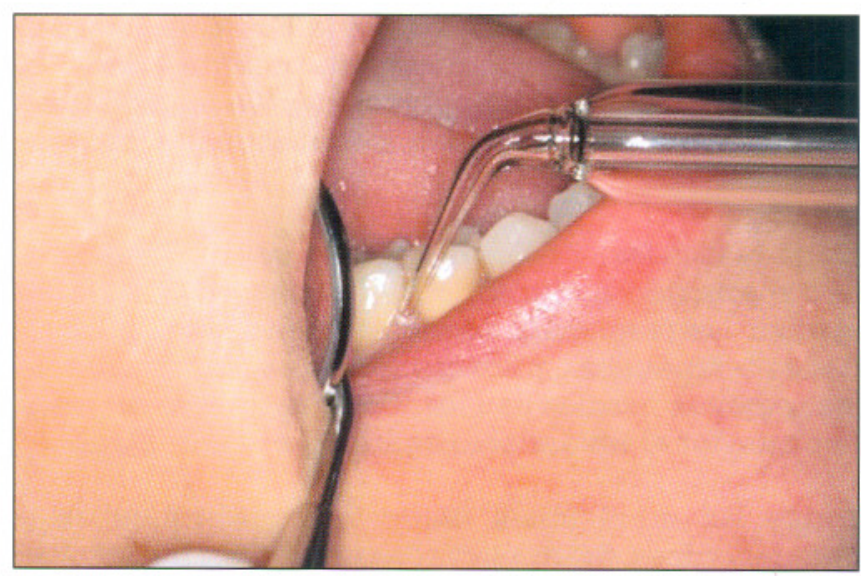

Imagen 9. Aplicación del ozono con PrimoLog $\mathrm{O}_{3}$ Sonda extrafina periodontal.

aplicación del tratamiento experimental se procedió a la aplicación del ozono a nivel del surco gingival con la cánula extrafina del sistema PrimoLogO ${ }_{3}$ de Kuss Dental (Figuras 7-10). Este sistema presenta varias canulas de aplicación para distintas especialidades 


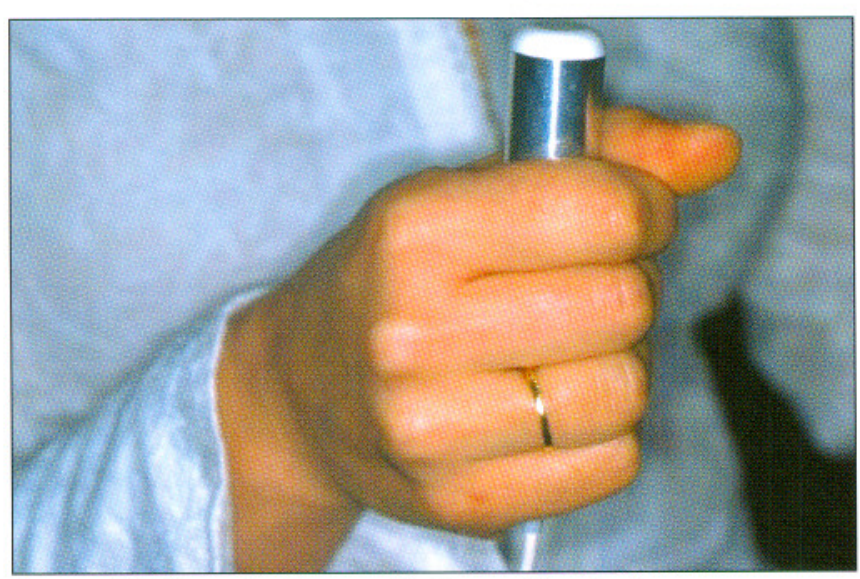

Imagen 10. Elemento de seguridad para el paciente.

Odontológicas, siendo la cánula extrafina la aplicada en el presente estudio. La intensidad inicialmente marcada para el estudio fue de cuatro. La mayoría de los pacientes fueron anestesiados por las hiperestesias producidas.

La aplicación del ozono fue limitada a 40 segundos por unidad de superficie dentogingival (vestibular, palatina o lingual)

La aplicación experimental del ozono fue realizada en tres ocasiones de forma consecutiva y en días alternos.

\section{RASPADO Y ALISADO RADICULAR: TRATAMIENTO GRUPO CONTROL.}

En la cuarta visita se procedió a la realización de una técnica de raspado y alisado radicular convencional para la eliminación mecánica de la bolsa periodontal y constituirán el grupo-cuadrante control contra el que se realizarán las comparaciones de las variables respuesta estudiadas en ambas modalidades terapéuticas.

Llegada esta fase clínica del ensayo se cita al paciente transcurrido un periodo de 30 dias $+/-5$, a fin de evaluar las respuestas producidas en ambas modalidades terapéuticas, permitiendo una comparación de los registros basales con los tomados en este momento posterior al tratamiento control y experimental.

Todas las mediciones clínicas, microbiológicas e inmunológicas, fueron llevadas a cabo en las mismas localizaciones periodontales realizadas en el estadio basal como en el postratamiento.

Tras el análisis y recopilación de todos los parámetros objeto de nuestro estudio, se procedió a la realización de un raspado y alisado radicular en aquellos cuadrantes, que actuaron como experimentales.

Metodología analítica. (Tablas 1-4) (Gráficos 1-7)

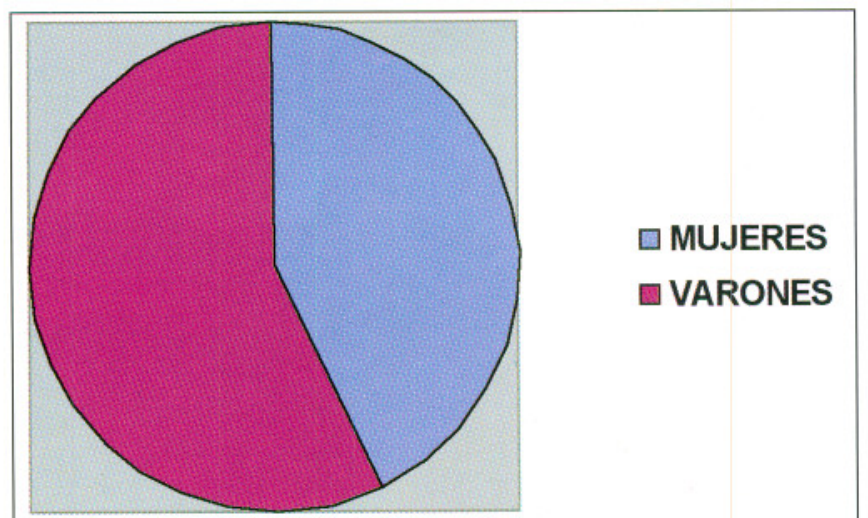

Gráfico 1. Distribución por sexos de la muestra poblacional.

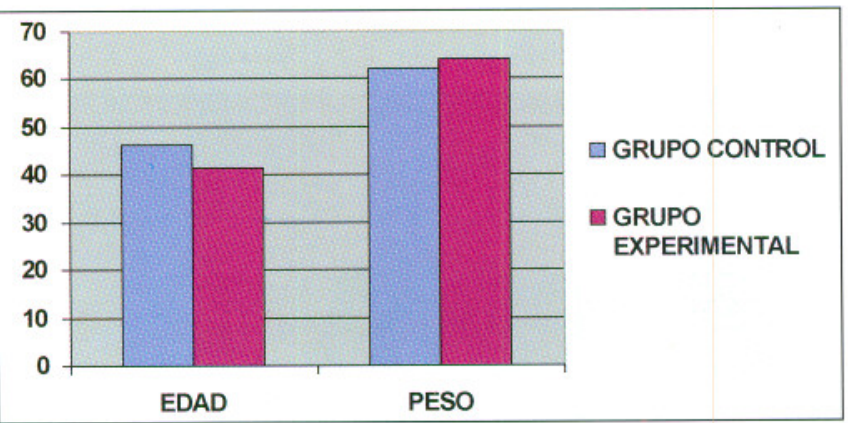

Gráfico 2. Distribución (Edad/Peso) de la muestra poblacional.

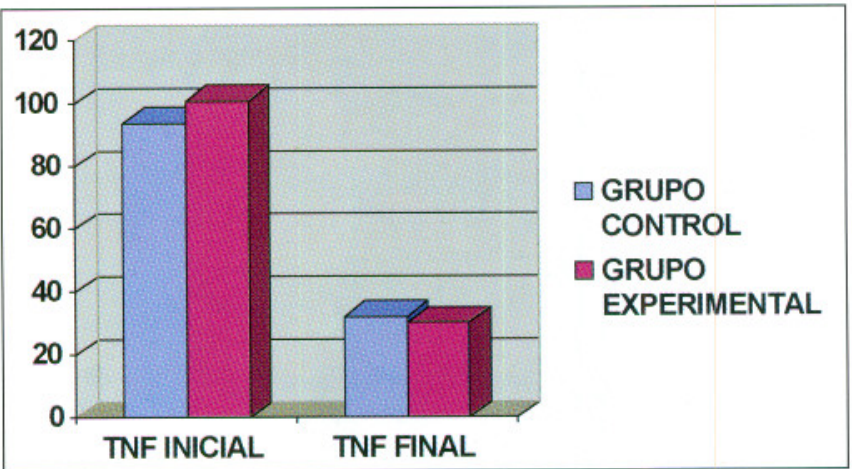

Gráfico 3. Concentración de TNF-alfa inicial y final de la muestra poblacional.

Las variables cuantitativas y continuas de nuestro estudio fueron valoradas mediante un análisis de la varianza bifactorial de medidas repetidas tiempo-tratamiento con el factor de repetición el tiempo y eliminando en el modelo estadístico la posible influencia del sujeto.

\section{RESULTADOS}

Los datos obtenidos se valoraron de forma preliminar mediante un análisis estadístico descriptivo en el que se indica que la población estudiada referenciada por 


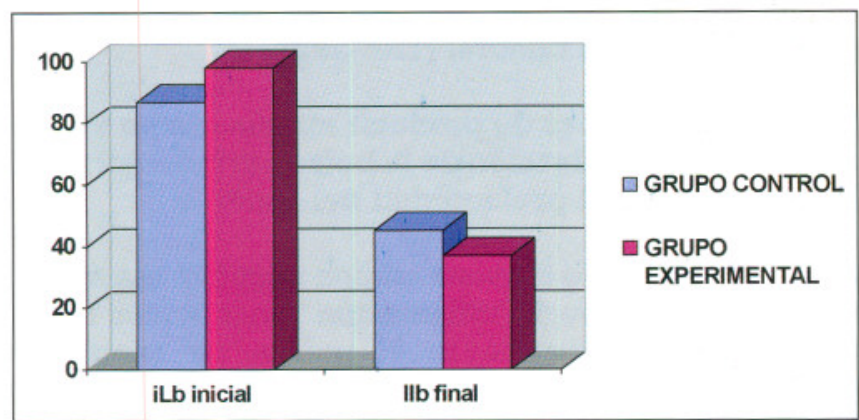

Gráfico 4. Concentración de Il-1b inicial y final de la muestra poblacional.

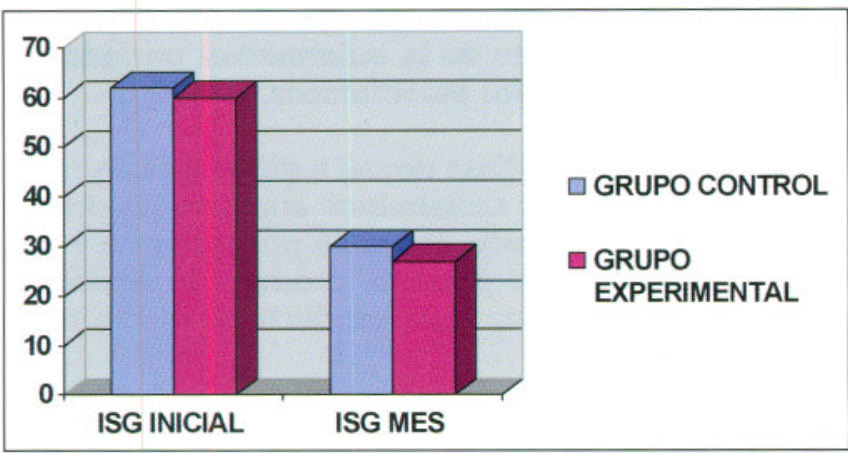

Gráfico 5. Indice de sangrado gingival inicial y final de la población.

edad y peso presenta una edad media de 48 y 42 años en los grupos-cuadrante control y experimental respectivamente. (Gráfico l y 2)

\section{Clínica}

Indice de sangrado gingival. En el grupo control el ISG fue> al 50\% de forma basal, tras la aplicación de ambas modalidades terapéuticas sufrió una reducción en términos medios del $50 \%$ sin existir una discrepancia estadísticamente significativa entre ambas modalidades terapéuticas. $(p<0,001)$ (Gráfico 5)

Indice de Higiene. Se produjo una reducción del índice de placa superior al $25 \%$ en ambos cuadrantes. Indice PI de Russell. (Gráfico 6)El grupo control presentó un puntuación próxima al 6 (periodontitis establecida) variando ésta en términos medios a una puntuación de 1,50 (gingivitis leve) tras la aplicación del tratamiento control. En el grupo experimental no se produjo variación alguna estadísticamente significativa; sin embargo se produjo una variación estadísticamente significativa entre ambas modalidades terapéuticas con una $\mathrm{p}<0,0005$.

\section{Microbiología}

Flora patógena periodontal total. El grupo control presentó una reducción significativa de su volumen microbiológico periodontopatógeno (Gráfico 7) de $36,4 \%$ siendo superior al $50 \%$ en el grupo experimental .El análisis de la varianza permitió destacar la

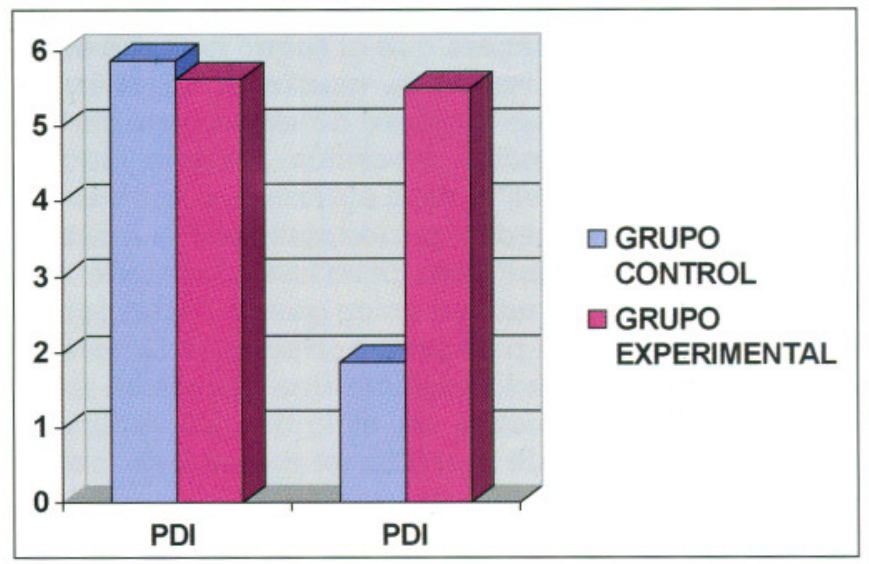

Gráfico 6. Indice PDI. Inical-final de la población.

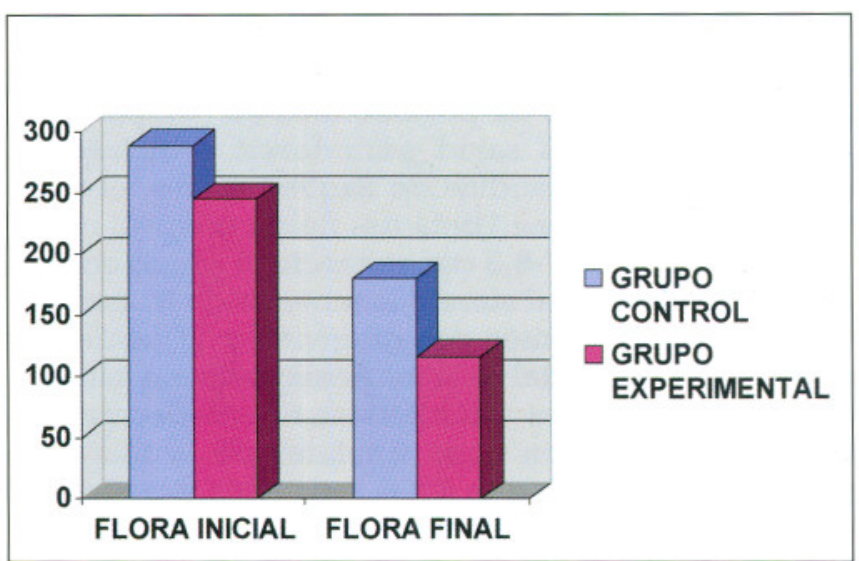

Gráfico 7. Periodontopatógenos totales inicio y final. Población de estudio $\left(\times 10^{5}\right)$

existencia de diferencia estadística significativa entre ambas modalidades terapéuticas con un $\mathrm{p}<0,269$.

\section{Inmunología.}

Inmunología: Il lb y TNF-alfa (Gráfico 3 y 4 ) fueron hallados en un porcentaje volumétrico inferior en ambos grupos control-experimental sin diferencia estadística significativa entre ambas modalidades con $\mathrm{p}<0,05$ (ANOVA).

\section{DISCUSIÓN}

El uso del ozono, con fines terapéuticos en otras especialidades médicas (traumatología, reumatología, angiología) entre otras, ha sido notablemente estudiado y aplicado con gran éxito en la actualidad; sin embargo su estudio en Odontología y Periodoncia es más limitado.

Desde un punto de vista clínico, se pudo observar como ciertos parámetros, tales como el índice de sangrado gingival se redujo significativamente de forma contraria a como lo hizo la profundidad de sondaje 
periodontal, de tal manera que el ozono no tiene efecto en relación a la remoción mecánica de la bolsa periodontal, dado que requiere de algún instrumento que permita su remoción mecánica. Por otro lado, la mayoría de los autores ${ }^{4,5,6}$ de la literatura, coinciden en relacionar la enfermedad periodontal con la existencia de un factor etiológico primario y considerado incluso por algunos autores único que se corresponde con la microbiología patógena periodontal. En base a este postulado se pudo explicar una reducción estadísticamente significativa en el índice de sangrado gingival35, puesto que la acción terapéutica del ozono es significativamente efectiva a nivel de los periodontopatógenos, permitiendo una disminución brusca de su concentración periodontal.

Interleuquina lbeta y TNF-alfa del fluido gingival crevicular $(\mathrm{GCF})^{36-41}$ son significativamente más elevados en todas las formas de periodontitis en comparación con los estados de salud periodontal o incluso de gingivitis. En los estudios de Saglie ${ }^{41}$ y cols, el volumen de interleuquina lbeta del fluido gingival crevicular fue de $16,8+/-5,3$ con respecto al grupo control de salud periodontal siendo la muestra de 21 pacientes sanos, 22 pacientes con gingivitis y 17 pacientes con periodontitis del adulto. Analizando en nuestro estudio las variables volumétricas de interleuquina y en comparación con otros similares de la literatura podemos establecer que dicha reducción significativa en ambas modalidades terapéuticas conduce a un estado de mejoría periodontal desde un punto de vista inmunológico. El mecanismo por el que el ozono actúa inmunológicamente ${ }^{30-1}$ a nivel periodontal lo desconocemos en la actualidad, siendo probable de gran interés para estudios mediante técnicas de marcación directa.

Analizando los datos clínicos referidos al índice de higiene pudimos observar una mejoría próxima al $25 \%$ en ambos grupos control-experimental, explicación que pensamos atribuir a la conciencia del propio paciente, al ser incluido en un programa de salud periodontal así como de una mayor motivación y educación para la salud.

\section{CONCLUSIONES}

En vista de los datos obtenidos, y de forma preliminar, podemos establecer unas conclusiones relativas a la ozonoterapia a nivel periodontal aplicadas a través del sistema PrimoLogO ${ }_{3}$ de la casa Kuss-Dental.

1.- El ozono, presenta un alto poder oxidativo a nivel periodontal, permitiendo una eficacia controlada en cuanto a parámetros de microbiología patógena periodontal e inmunología (interleuquina l-beta y factor de necrosis tumoral (TNF-alfa).

2.- No ha sido capaz de producir ningún efecto mecánico sobre la eliminación de la bolsa periodontal ni en la reducción de la profundidad del sondaje.

3.- Desde un punto de vista clínico permite una mejoría significativa en la inflamación periodontal reduciendo significativamente el índice de sangrado gingival

4.- A nuestro parecer este novedoso estudio permite abrir un nuevo campo de investigación en Odontología y en Periodoncia para favorecer nuevas alternativas clínicas en el tratamiento de la enfermedad periodontal en sus distintos estadios patocrónicos.

5.- $\mathrm{El} \mathrm{PrimoLog} \mathrm{O}_{3}$ de Kuss dental a diferencia de otros aparatos y siestemas actualmente comercializados es en la actualidad el único, capaz de por si mismo y con el propio oxígeno del paciente producir la oxidación periodontal necesaria para producir su efecto terapéutico.

\section{ABSTRACT}

INTRODUCTION. In recent years, important progress has been made in the knowledge of periodontal disease, from a multifactorial etiology, the responsable microbiology, the local inmunological response as also multiple aspects related to its epidemiology, etiology and its evolution. Given a greater knowledge in thsese fields new techniques (pharmacological products and apparatus) are being developed to treat and detain the periodontal disease and preserve dental health.

OBJECTIVES. Analyse the periodontal response from a clinical, microbiological and immunological point of view of a population with moderate-severe periodontal pockets and its comparison with the scraping and smoothing periodontal technique.

METHOD AND MATIRIAL. A total of 72 cuadrants with at least more than four teeth in each cuadrant and more than $6 \mathrm{~mm}$. pocket depth probe were studied in a population of 43 average age. To establish comparison between both groups a cross mouth study has been done, in which half were treated with ozone and the rest with scraping technique. The variable results were evaluated: clinical, microbiological and immunological response with descriptive estadistic analysis and ANOVA.

CONCLUSION: We can say that the periodontal treatment with ozone produces estadisticaly significant reduction in the amount of index gingival bleeding, in the microbiological periodontal parameters as also in the immunological patterns of ILlb y TNF-alfa, howe- 
ver there is no variation in relation to the level periodontal insertion and the depth of the periodontal pocket that requires a periodontal technique of scraping.

\section{KEY WORDS}

Ozone, Microbiology, Inmunology, Periodontal disease, Biooxidation.

\section{CORRESPONDENCIA}

Jorge Ripollés de Ramón.

C/ Marbella, 68-5ㄴ

28034 Madrid

654872716@amena.com.

Tel.: 654872716 ó 917348305.

\section{BIBLIOGRAFÍA}

1. Arano JM, Izarbe LM. Propuesta experimental para el mantenimiento en periodoncia mediante oxigenoterapia. Gac Den. 1999; Nov (102): 36-42.

2. Izarbe LM. Ozono: generalidades. Aplicaciones en Medicina y Odontología. Maxillaris 2000; (18): 36-42.

3. Brauner A. Clinical studies of therapeutic results from ozonized water for gingivitis an peiodontitis. J Periodon. 2001;13:24-7.

4. Bascones Martínez A. Etiopatogenia de las enfermedades periodontales. Tratado de Odontología. 1999. Tomo III-XXVII (2): 3319-27.

5. Echeverría García JJ. Epidemiología y clasificación de las enfermedades periodontales. Manual de Odon-tología. 1995 Masson. España.

6. Lindhe J. Periodontología clínica. Editoraial Panamericana. 1992: 70-140.

7.Zambon JJ. Actinobacillus actynomicetemcomitans in human periodontal disease. J. Clin.Periodontol. 1985; 12:1

8. Socransky SS. Microbiology of periodontal disease. J. Clin. Periodontol. 1977;48:497.

9. Listgarten MA. Structure of microbial flora asocciated with periodontal health and disease in man. A light and electron microscopic study. J. Periodontol. 1976; 47: 1-3.

10. Offenbacher S Costopoulus SV. Microbial colonization patterns of loosely adherent subgingival plaque in adult periodontitis. J. Clin. Periodontol. 1988; 15: 53-9.

11. Slots J, Taubman M. Contemporary oral microbiology and inmunology. $1^{\text {a }}$ Ed. Mosby. 1992.
12. Bascones A. Periodoncia Diagnóstico y Tratamiento de la enfermedad periodontal. $3^{a}$ Ed. Avances. 145-184. Madrid.1991.

13. Ramfjord SP. Indices for prevalence and incidence of periodontal disease. J. Periodontal Disease. J. Perio-dontol. 1959: 30: 51-9.

14. Russell AL A system of classification and scoring for prevalence surveys of periodontal disease. J. Dent. Res. 1956; 35: 350-9.

15. O'Leary TJ. The periodontal screening examination. J. Periodontol. 1967: 38: 617-24.

16. O'Leary TJ, Drake RB, Naylor JE. The plaque control record. J Peridontol. 1972; 43: 38-46.

17. Löe $\mathrm{H}$. The gingival index, Plaque index and retention index systems. J. Periodontol 1967: 38: 610-6.

18. Löe H, SilnessJ. Periodontal disease in pregnancy, Prevalence and severity. Acta Odont Scandinav. 1963; 21 : 533-51.

19. Sas/Stat, User's Guide, vestats cary NC. SAS Institute. Inc. $1999 ; 3884$.

20. Zambon JI Actinobacillus actinomycetemcomitans in human periodontal disease prevalence in patient group and distribution of byotipes and serotypes within families. J. Periodontol. 1983; 54: 704-12.

21. Minguez F. Antimicrobial activity of ozonized water in determined experimental conditions. Rev Sanid Hig Publica. 1990; (64): 415-23.

22. Velano HE. In vitro assesment of antibacterial activity ozonized water against Staphylococcus aureus.Pesqui Odontol Bras. 2001; 15 (1): 18-22.

23. Moore G Griffith C, Peters A. Bactericidal Properties of ozone and its potencial application as a terminal desinfectant. J. Food Prot. 2000.63 (8): 1100-6.

24. Secgi LA, Lezcano I. Antibacterial activity of ozonized sunflower oil. J. Appl. Microbiol.2001; 90 (2): 279-84.

25. Figueredo CM, Ribero MS. Increased interleukin lb concenmtration in gingival crevicular fluid as a characteristic of periodontitis. J. Periodontol. 1999: 70: 1457-63.

26. Ebersole JL. Gingival crevicular fluid inflamatory and bacteriology of gingivitis in nonhuman primates related to susceptibility to periodontitis. Oral Microbiolo. Inmunol. 2000. 15: 19-26.

27. Gamonal J, Acevedo A, Bascones A, Jorge O, Silva A. Levels of interleukin $1 \mathrm{~b}-8$ and 10 and RANTES in gingival crevicular fluid and cell popullation in adult periodontitis patients and the effect of periodontal treatment. J. Periodontol 2000: 71; 1531-45.

28. Garrison SW, Holt SC. Lypopolysccharides prepared from suspected periodontal pathogens. J. Periodontal 1998: 59: 684.7 


\section{AVANCES}

Volumen 16 - No 1 - Abril 2004

29. Haffajee AD, Socranski E, Mooney J. Clinical Microbiological and inmunological features of subjects eith destructuive periodontal disease. J. Clin. Periodontol. 1998: $15 ; 240-6$.

30. Ishira Y. Gingival crevicular Interlekinl and 1 receptor against levels in periodontally healthy and disease sites. J. Periodontal Res. 1997: 32: 524-9.

31. Jin L. Interleukin-8 and granulocite elastase in gingival crevicular fluid in relation to periodontophatogens in untreated adult periodontitis. J. Periodontol. 2000: 71: 929-39.

32. Offenbacher S. Periodontaldisease: Pathogenesis. Ann Periodontol 1996: 1: 821-78.

33. Page RC, Beck JD. Risk assesment for periodontal disease. Int. Dent.J. 1997: 47: 61-87.

34. Page RC. Pathogenesis of inflamatory periodontal disease. Lab Invest. 1976: 33: 235-9.

35. Wheeler TT, Mc Arthur WP. Modeling the relationship between clinical, microbiological and inmunologic parameters and alveolar bone levels in an elderly population. J. Periodontol. 1994; 65: 68-78.
36. Aarli V. Effet of venous stasys and hipoproteinemia in gingival fluid formation in rats. J. Periodontal Res. 1995; 30: 231-7.

37. Curtis MA. The total protein concentration of gingival crevicular fluid. Variation with sampling time and gingival inflamation. J. Clin.Periodontol. 1988: 15: 628-32.

38. Curtis. The protein composition of gingival crevicular fluid sample fom male adolescentes with no destructive perioodntitis. Baseline data of a longitudinal study. J. Periodontal Res. 1990: 25: 6-16.

39. Cimasoni G. Crevicular fluid update. Monograph in oral scines. Vol 12 Basel. Karger. 1983.

40. Pekovic DD, Fillery ED. Identification of bacteria in inmunophatological mechanism of human periodontal disease. J. Periodontal Res. 1984: 19: 329-51.

41. Saglie R. Bacterial invasión of gingiva in advanced periodontitis in humans. J. Periodontol 1982: 53: 217-22. 